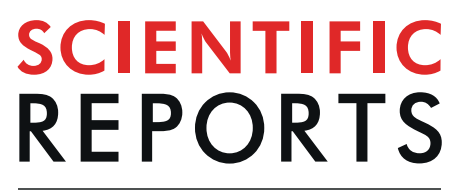

natureresearch

Corrected: Author Correction

\title{
Macrophage Mannose Receptor CD206 Predicts Prognosis in Community-acquired Pneumonia
}

\begin{abstract}
Kazuo Tsuchiya ${ }^{1,3}$, Yuzo Suzuki iD ${ }^{1,3 *}$, Katsuhiro Yoshimura ${ }^{1}$, HidekiYasui ${ }^{1}$, Masato Karayama ${ }^{1}$, Hironao Hozumi ${ }^{1}$, Kazuki Furuhashi ${ }^{1}$, Noriyuki Enomoto ${ }^{1}$, Tomoyuki Fujisawa ${ }^{1}$, Yutaro Nakamura ${ }^{1}$, Naoki Inui ${ }^{1}$, Koushi Yokomura ${ }^{2}$ \& Takafumi Suda $^{1}$

CD206, a mannose receptor, is mainly expressed on the surface of alternatively activated macrophages where it acts as a pattern recognition receptor and plays a role in innate and adaptive immunity. This study investigated serum soluble CD206 (sCD206) levels in community-acquired pneumonia (CAP) and examined their clinical significance. SCD206 concentrations were measured in the sera of two independent cohorts with CAP (127 and 125 patients, respectively) and 42 controls. The expression of CD206 in the lung from autopsied cases was also examined. Patients with CAP showed significantly elevated sCD206 levels than did the controls $(p<0.0001)$. Notably, fatal CAP patients had more than two-fold higher sCD206 concentrations than survivors in both cohorts $(p<0.0001)$. Serum sCD206 concentrations were associated with Pneumonia Severity Index (PSI) and CURB-65 values. Importantly, even fatal CAP patients classified as PSI I-IV, CURB65 0-2 or age $<75$ years had comparatively higher levels of SCD206 than those classified as PSI V, CURB-65 3-5 or age $\geq 75$ years. Immunohistochemically, the infiltration of $\mathrm{CD}^{206}{ }^{+}$macrophages was found in the lungs of fatal cases. Elevated levels of sCD206 are associated with CAP prognosis, suggesting SCD206 might be a potential biomarker to predict severity for CAP.
\end{abstract}

Community-acquired pneumonia (CAP) is the most common infectious disease and a leading cause of morbidity and mortality worldwide ${ }^{1}$. The pathogenesis of infectious disease varies based on the causative agent involved, as well as host immune status, which in turn, is regulated via a complex mechanism. Inflammation is an essential defense mechanism at the mucosal level, wherein immune cells are activated in response to invading pathogens. At the same time, immunosuppression or immune tolerance protects the host from excessive or inappropriate immune activation. Macrophages have a pivotal role in the innate immune response, and phenotypically-polarized macrophages are generally classified into two major subtypes, namely pro-inflammatory M1 and anti-inflammatory M2 macrophages ${ }^{2,3}$. We previously demonstrated that soluble CD163 and indoleamine 2,3-dioxygenase (IDO), an immunoregulator produced by macrophages and dendritic cells, are associated with disease severity and the prognosis of $\mathrm{CAP}^{4,5}$. This suggests that immunomodulatory molecules can potentially serve as surrogate markers for infectious diseases.

CD206 is a mannose receptor expressed on alternatively activated macrophages, termed M2 macrophages. M2 macrophages have an anti-inflammatory phenotype to resolve excess inflammation, promoting wound healing and also inducing immunotolerance ${ }^{2,3}$. On the surface of macrophages, CD206 acts as a pattern recognition receptor (PPR) for various pathogens including viruses, fungi, and bacteria; further, this molecule recognizes their mannan-coated cell walls or envelopes, and functions following endocytosis, phagocytosis and antigen presentation $^{6-9}$. Additionally, polysaccharide from Streptococcus pneumoniae and lipopolysaccharide from Klebsiella pneumoniae bind CD206 ${ }^{10}$, indicating roles for these molecules in infectious disease. Upon proteolytic cleavage of the membrane-bound form, the soluble form of CD206 (sCD206) is produced. Importantly sCD206 also recognizes sulphated and mannosylated carbohydrates ${ }^{10,11}$. As this molecule can be identified at peripheral sites, we hypothesized that evaluating its levels might represent macrophage activity and could be a potential biomarker for CAP. Therefore, in this study, we measured sCD206 levels in patients with CAP and evaluated their clinical implications. We also examined membrane-bound CD206 in the lungs of autopsied cases by immunohistochemistry.

\footnotetext{
${ }^{1}$ Second Division, Department of Internal Medicine, Hamamatsu University School of Medicine, Hamamatsu, Japan. ${ }^{2}$ Department of Respiratory Medicine, Seirei Mikatahara Hospital, Hamamatsu, Japan. ${ }^{3}$ These authors contributed equally: Kazuo Tsuchiya and Yuzo Suzuki. *email: yuzosuzu@hama-med.ac.jp
} 


\begin{tabular}{|c|c|c|c|}
\hline & Cohort1 $(n=127)$ & Cohort2 $(n=125)$ & $p$-value \\
\hline Sex, M/F & $77 / 50$ & $77 / 48$ & 0.898 \\
\hline Age, years & $73.0(21-99)$ & $76.0(31-93)$ & 0.006 \\
\hline \multicolumn{4}{|l|}{ Comorbidities } \\
\hline Congestive heart failure (n, \%) & $23(18.1)$ & $15(12.0)$ & 0.218 \\
\hline Chronic pulmonary disease $(\mathrm{n}, \%)$ & $47(37.3)$ & $66(52.8)$ & 0.016 \\
\hline Renal disease $(\mathrm{n}, \%)$ & $4(3.1)$ & $17(13.6)$ & 0.003 \\
\hline Diabetes mellitus (n, \%) & $11(8.7)$ & $33(26.4)$ & $<0.001$ \\
\hline Chronic liver disease $(\mathrm{n}, \%)$ & $5(3.9)$ & $11(8.8)$ & 0.128 \\
\hline Cerebrovascular disease $(\mathrm{n}, \%)$ & $23(18.1)$ & $11(8.8)$ & 0.042 \\
\hline Neoplastic disease (n, \%) & $12(9.4)$ & $38(30.4)$ & $<0.001$ \\
\hline Immunosuppressive agents (n, \%) & $7(5.6)$ & $33(26.4)$ & $<0.001$ \\
\hline \multicolumn{4}{|l|}{ Clinical characteristics } \\
\hline Body temperature, & $37.8(35.0-40.8)$ & $37.8(35.6-40.3)$ & 0.462 \\
\hline Systolic blood pressure $<90 \mathrm{mmHg}(\mathrm{n}, \%)$ & $7(5.5)$ & $7(5.6)$ & 1.000 \\
\hline Confusion (n, \%) & $24(19.0)$ & $12(9.6)$ & 0.047 \\
\hline Respiratory Failure $\left(\mathrm{SaO}_{2}<90 \%\right)(\mathrm{n}, \%)$ & $64(50.8)$ & $74(59.2)$ & 0.205 \\
\hline $\mathrm{PaO}_{2} / \mathrm{FiO}_{2}$ ratio & $291.00(49.8-428.60)$ & $260.95(42.1-496.43)$ & 0.006 \\
\hline \multicolumn{4}{|l|}{ Laboratory findings } \\
\hline BUN (mg/dL) & $16.70(4.5-206.0)$ & $20.00(7.3-172.5)$ & 0.008 \\
\hline Cre (mg/dl) & $0.75(0.23-6.69)$ & $0.87(0.38-10.60)$ & 0.003 \\
\hline $\mathrm{Alb}(\mathrm{g} / \mathrm{dl})$ & $3.50(2.30-5.00)$ & $3.10(1.80-4.50)$ & $<0.001$ \\
\hline $\mathrm{WBC}(/ \mu \mathrm{L})$ & $10760(2300-37000)$ & $10170(1000-29330)$ & 0.932 \\
\hline CRP (mg/dl) & $12.00(0.17-57.65)$ & $12.04(0.09-50.17)$ & 0.886 \\
\hline PCT (ng/ml) & ND & $0.68(0.02-87.8)$ & NA \\
\hline Bacteremia & $8(6.3)$ & $7(5.6)$ & 0.136 \\
\hline
\end{tabular}

Table 1. Clinical characteristics of patients with community acquired pneumonia. BUN; blood urea nitrogen, Cre; creatinine, Alb; albumin, CRP; C-reactive protein, PCT; procalcitonin, $\mathrm{P} / \mathrm{F}$ ratio; $\mathrm{PaO}_{2} / \mathrm{FiO}_{2}$ ratio. Data are shown by median (minimum-maximum).

\section{Results}

Clinical characteristics. Clinical characteristics of 127 patients with CAP in Cohort 1 and 125 patients in Cohort 2 are summarized in Table 1 . There were no obvious differences in sex, but Cohort 2 comprised older individuals than Cohort1. The presence of respiratory failure $\left(\mathrm{SaO}_{2}<90 \%\right.$ or $\mathrm{PaO}_{2}<60$ Torr $)$ or impaired consciousness was not differed between the cohorts. Most patients showed elevated levels of C-reactive protein (CRP) and increased white blood cell counts. Serum levels of procalcitonin (PCT) were measured for 78 patients in Cohort2, of which 45 showed positive PCT (cut-off, $0.5 \mathrm{ng} / \mathrm{ml}$ ). Blood cultures were positive for samples from eight patients $(6.3 \%)$ in Cohort1 and seven patients $(5.6 \%)$ in Cohort2.

The severity and outcomes of CAP patients are presented in Table 2. The median Pneumonia Severe Index (PSI) was 91 points in Cohort1 and 107 points in Cohort2, respectively, indicating that severity tended to be higher in Cohort $2(\mathrm{p}=0.022)$, and more than $50 \%$ of patients were categorized in PSI class $\geq$ IV. The median CURB-65 score was 1.0 in each cohort, and this score indicated higher severity in Cohort2 $(\mathrm{p}=0.032)$; approximately half of patients were classified as CURB-65 Class $0-1$. The proportions of CAP patients requiring mechanical ventilation or ICU administration were not different between the cohorts. Overall mortality due to respiratory failure or multiple organ failure associated with CAP after admission was 10.2\% (13 patients) in Cohort 1 and $7.2 \%$ (nine patients) in Cohort 2 but did not differ between the cohorts. The identified pathogens were showed in the Supplement Table 1.

Serum concentrations of sCD206 in Cohort1. The serum concentrations of sCD206 in CAP patients in Cohort1 are presented in Fig. 1. The mean serum level of sCD206 in patients with CAP was $718.8 \mathrm{ng} / \mathrm{ml}$ [90.38-4035.55], whereas that in healthy controls was $329.2 \mathrm{ng} / \mathrm{ml}$ [104.87-660.92], indicating significantly elevated levels in patients with CAP $(\mathrm{p}<0.0001$, Fig. $1 \mathrm{~A})$. Further, the patients who died had significantly higher levels of serum sCD206 (1644 ng/ml [340.41-3130.15]) compared to those in survivors (683 ng/ml [90.38-4035.55], $\mathrm{p}<0.0001$; Fig. 1B).

Serum concentrations of sCD206 in Cohort2. We separately evaluated sCD206 levels in patients with CAP in another cohort. In Cohort2, similar to that in Cohort1, patients with CAP had significantly higher levels of sCD206 than controls (1086 ng/ml [118.155-4188.05] vs $329.2 \mathrm{ng} / \mathrm{ml}$ [104.87-660.92], p < 0.0001, Fig. 1A). Additionally, CAP patients who died had more than two-fold higher levels of sCD206 compared to those in surviving CAP patients (2232 ng/ml [954.35-4188.05] vs. 1058 ng/ml [118.155-2923.375], p < 0.0001; Fig. 1B). 


\begin{tabular}{|c|c|c|c|}
\hline & Cohort1 $(n=134)$ & Cohort2 $(n=125)$ & $p$-value \\
\hline \multicolumn{4}{|l|}{ PSI, no (\%) } \\
\hline I & $15(11.8)$ & $3(2.4)$ & \multirow{5}{*}{0.022} \\
\hline II & $18(14.2)$ & $13(10.4)$ & \\
\hline III & $30(23.6)$ & $27(21.6)$ & \\
\hline IV & $40(31.5)$ & $51(40.8)$ & \\
\hline $\mathrm{V}$ & $24(18.9)$ & $31(24.8)$ & \\
\hline \multicolumn{4}{|l|}{ CURB65, no. (\%) } \\
\hline $0-1$ & $71(55.9)$ & $65(52.0)$ & \multirow{3}{*}{0.032} \\
\hline 2 & $27(21.3)$ & $43(34.4)$ & \\
\hline $3-5$ & $29(22.8)$ & $17(13.6)$ & \\
\hline $\operatorname{IPPV}(\mathrm{n}, \%)$ & $11(8.7)$ & $5(4.0)$ & 0.195 \\
\hline $\operatorname{NPPV}(n, \%)$ & $5(3.9)$ & $8(6.4)$ & 0.409 \\
\hline ICU admission (n, \%) & $12(9.4)$ & $9(7.2)$ & 0.650 \\
\hline Duration of hospitality & $14.5(0-149)$ & $15(2-75)$ & 0.189 \\
\hline Mortality (n, \%) & $13(10.2)$ & $9(7.2)$ & 0.504 \\
\hline
\end{tabular}

Table 2. Severity and Outcome of patients with community acquired pneumonia. IPPV; invasive positive pressure ventilation, NPPV; non-invasive positive pressure ventilation, ICU; intensive care unit. Data are shown by median (minimam-maximam).

(A)

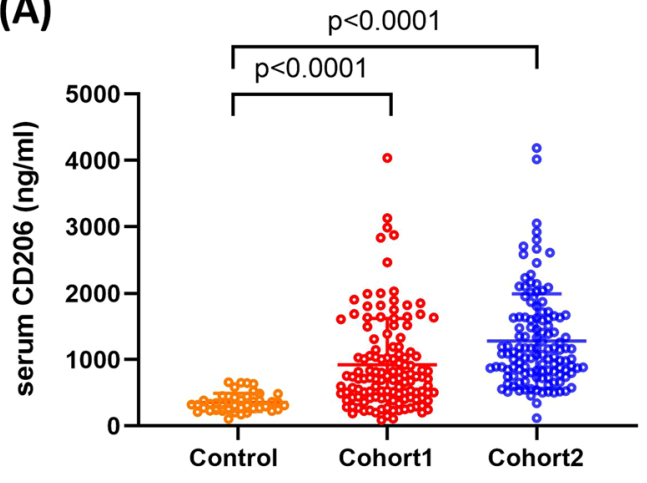

(B)

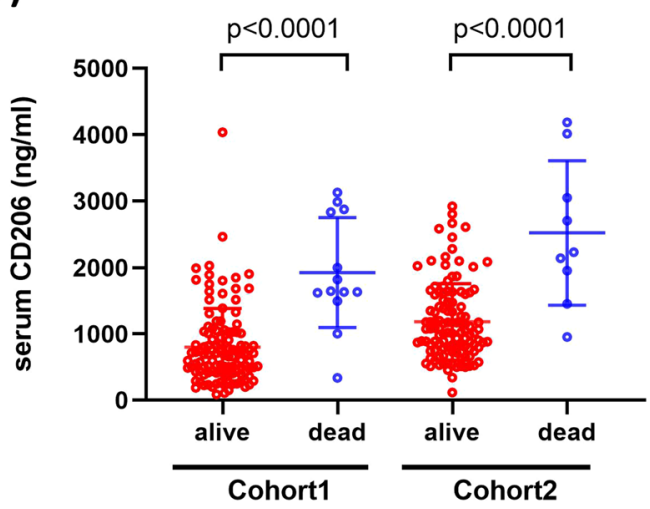

Figure 1. Serum concentrations of sCD206 in patients with community-acquired pneumonia (CAP). Serum concentrations of sCD206 in patients with CAP and control subjects (A), and in CAP patients with nonsurvivors and survivors (B). P-values were determined by Mann-Whitney $\mathrm{U}$ test.

Correlations between sCD206 and disease severity or clinical parameters. We next evaluated serum sCD206 levels according to CAP severity using PSI and CURB-65 scales. As shown in Fig. 2A,B, serum concentrations of sCD206 were increased according to severity of the CAP index. Additionally, correlation analyses showed that sCD206 was positively correlated with PSI score and CUB-65 score, as well as BUN and CRP levels, but was negatively associated with $\mathrm{PaO}_{2} / \mathrm{FiO}_{2}$ ratio (P/F ratio) and albumin levels (Supplement Table 2).

sCD206 levels in patients and non-survivors according to disease severity and age. Although a significantly higher mortality rate was found for CAP patients classified into PSI V $(\mathrm{p}=0.001)$, CURB-65 $3-5(\mathrm{p}=0.001)$ and age $\geq 75$ years groups, more than $40 \%$ of the non-surviving patients were classified as PSI $<\mathrm{V}(\mathrm{n}=10,45.5 \%)$, CURB$65<3-5(\mathrm{n}=11,50 \%)$, and age $<75$ years $(\mathrm{n}=10,45.5 \%)$. Thus, we next evaluated sCD206 levels in 22 patients compared to those in non-survivors. The patients with CAP who died had significantly elevated serum sCD206 levels than those in surviving patients regardless of severity in terms of PSI index $(816.4 \mathrm{ng} / \mathrm{ml}[90.38-2806.85]$ vs. $1798.04 \mathrm{ng} / \mathrm{ml}$ [954.35-3050.13] for PSI I-IV as compared to $1072.62 \mathrm{ng} / \mathrm{ml}$ [200.64-4035.55] vs. $2116.15 \mathrm{ng} / \mathrm{ml}$ [340.41-4188.05] for PSI V; $\mathrm{p}<0.0001$ and $\mathrm{p}=0.0028$, respectively, Fig. $3 \mathrm{~A})$, and CURB65 index $(835.85 \mathrm{ng} / \mathrm{ml}$ [90.38-2806.85] vs. $1951.77 \mathrm{ng} / \mathrm{ml}$ [954.35-3130.15] for PSI I-IV as compared to $1010.1 \mathrm{ng} / \mathrm{ml}$ [200.64-4035.55] vs. $2000.06 \mathrm{ng} / \mathrm{ml}$ [340.41-4188.05] for PSI V; $\mathrm{p}<0.0001$ and $\mathrm{p}=0.0050$, respectively, Fig. 3B). The patients with CAP who died had significantly elevated serum sCD206 levels in both age groups $(741.15 \mathrm{ng} / \mathrm{ml}[90.38-4035.55]$ vs. $2261.3 \mathrm{ng} / \mathrm{ml}$ [1493.9-4188.05] for age $<75$ years as compared to $1005.81 \mathrm{ng} / \mathrm{ml}$ [118.155-2923.375] vs. $1975.92 \mathrm{ng} / \mathrm{ml}$ [340.41-3130.15] for age $\geq 75$ years; $\mathrm{p}<0.0001$ and $\mathrm{p}=0.0015$, respectively, Fig. 3C). Additionally, when comparing non-survivors, the levels of sCD206 were comparable between PSI I-IV and PSI V, CURB65 0-2 and CURB65 $3-5$, and age $<75$ years and age $\geq 75$ years. 
(A)

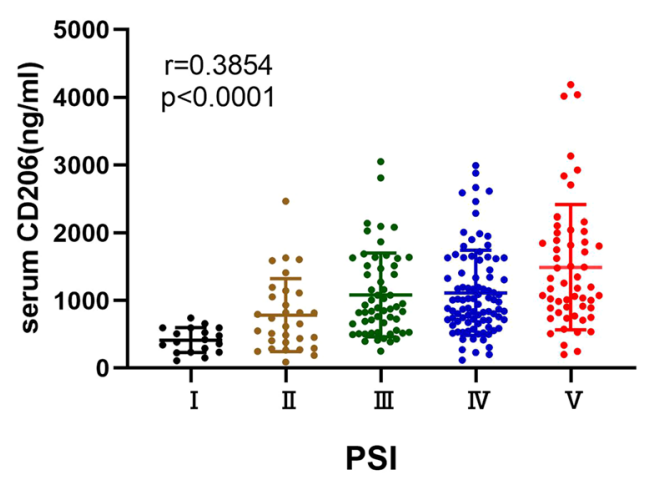

(B)

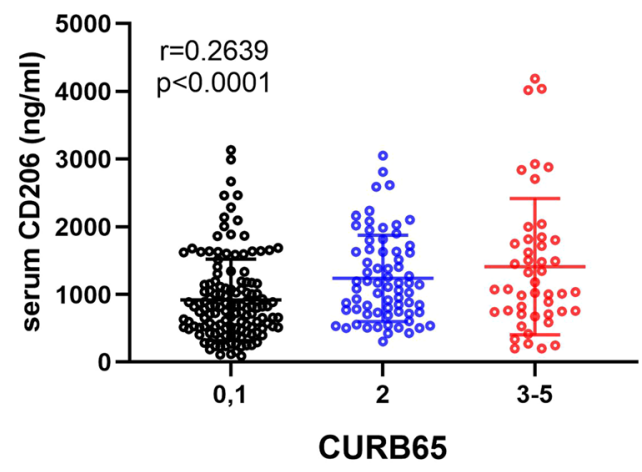

Figure 2. Serum concentrations of sCD206 in patients with community-acquired pneumonia (CAP) according to the disease severity. Serum concentrations of sCD206 in patients with CAP according to the PSI (A) and CURB65 (B). $P$-values were determined using the ANOVA test.

(A)

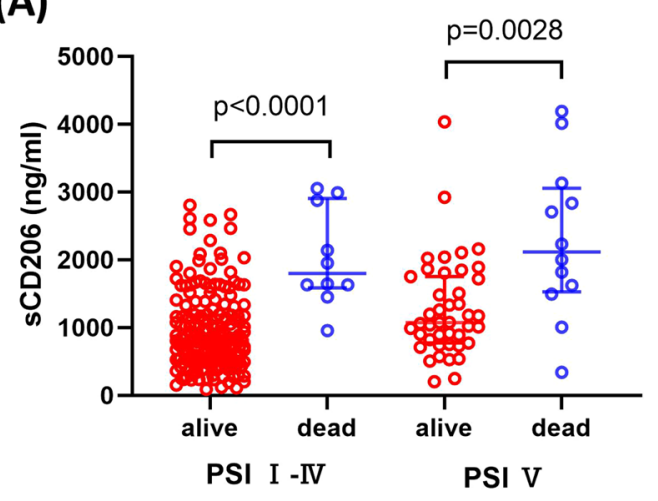

(C)

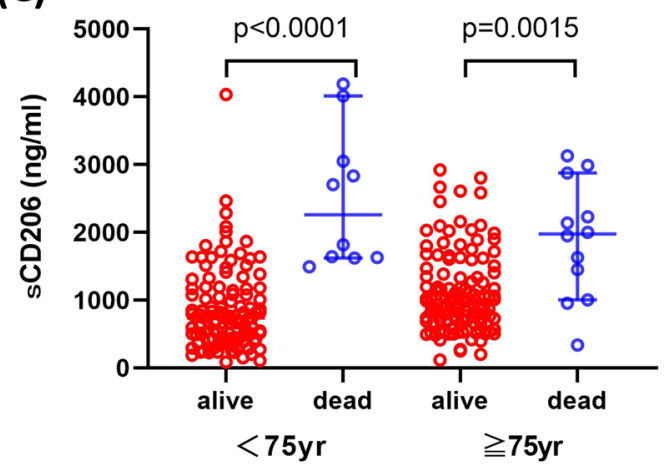

(B)

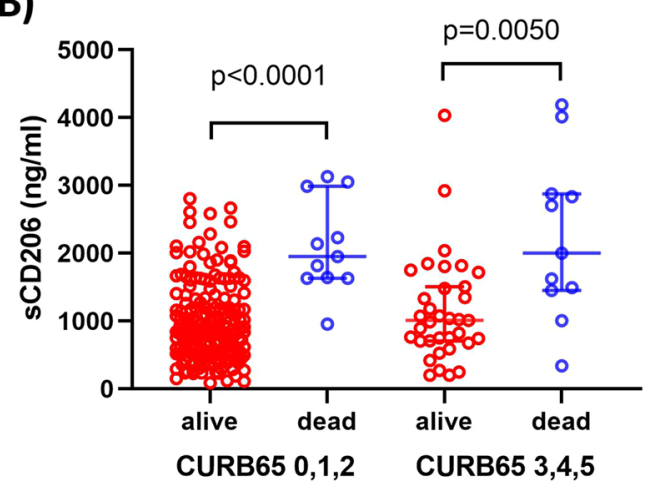

Figure 3. Serum concentrations of sCD206 in patients with community-acquired pneumonia (CAP) according to prognosis. Serum concentrations of sCD206 in patients with CAP according to prognosis classified by the PSI (A), CURB65 (B), and Age (C). P-values were determined by Mann-Whitney U test.

Univariate and multivariate analyses of prognostic factors. We next calculated the optimal cut-off values for SCD206 to predict death using ROC analysis. Based on the ROC analysis, the area under the ROC curve (AUC) was 0.856 for sCD206 (Fig. 4), which was superior to that for CRP, PSI, or CURB65 score (0.674, 0.770 , and 0.747 , respectively). The AUC for PCT was 0.603 for 78 patient samples. Using the optimal cut-off for serum sCD206 levels $(1413 \mathrm{ng} / \mathrm{ml})$, sensitivity and specificity values obtained were $86.4 \%$ and $79.1 \%$, respectively, with a likelihood ratio of 4.13 . We then assessed the prognosis of patients with CAP based on this cut-off value, using the Kaplan-Meier method and log-rank test. The high-sCD206 ( $n=67)$ group showed significantly lower survival rates than the low-sCD206 $(n=185)$ group $(\mathrm{p}<0.0001$; Fig. 5A). Although age-related scoring systems such as PSI and CURB65 are widely validated and confirmed ${ }^{12,13}$, certain situations have been associated with concerns about validity. For example, there are potential risks of overestimating severity in elderly patients and 


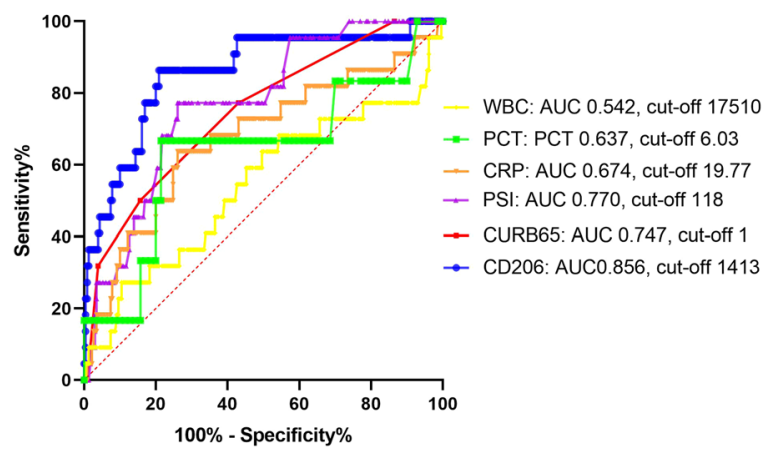

Figure 4. Receiver operator curve analysis for predicting mortality in patients with community-acquired pneumonia (CAP). Receiver operator curve analysis for predicting mortality in patients with CAP.

misclassifying younger patients with severe disease into lower classes ${ }^{14-16}$. Therefore, we performed a subgroup analysis of sCD206 according to patient age and confirmed that this cut-off worked, regardless of patient age (Fig. 5B,C). Finally, to determine the prognostic value of sCD206 with regard to outcome, we performed logistic regression analyses (Table 3). By univariate analysis and age/sex-adjusted multivariate analyses, sCD206, as well as PSI, CURB-65, and CRP, was found to be a significant predictor of death.

Expression of CD206 in the lung biopsies of patients with CAP. To investigate the involvement of M2 macrophages and sources of sCD206 in patients with severe CAP, we assessed CD206 expression in autopsied-lung tissues from fatal CAP patients by immunohistochemistry. An autopsied lung specimen from a 75-year-old male with fatal CAP showed dense infiltration of neutrophils and CD206 ${ }^{+}$macrophages with fibrinous exudates in the alveolar space (Fig. 6). Similarly, the accumulation of CD206-positive macrophages was found in the lungs of the remaining cases (Supplement Figs. 1,2). Meanwhile, only a few CD206-positive macrophages were found in the alveolar spaces in the resected lung sections from patient with early lung cancer (Supplement Fig. 3).

\section{Discussion}

In the present study, we measured the serum sCD206 levels in patients with CAP and evaluated their clinical implications using two independent cohorts. Compared with control subjects, we found that patients with CAP demonstrated a significant increase in serum sCD206 that is associated with disease severity and prognosis. The accumulation of $\mathrm{CD} 06^{+}$macrophages in the alveolar and interstitial spaces was also found in the lungs of autopsied cases. Importantly, we found that sCD206 is a potential surrogate marker to predict CAP outcome.

Macrophages are abundant cells in the lungs and have pivotal roles in first-line defense and the maintenance of homeostasis ${ }^{8,17}$. On the surface of macrophages, CD206 serves as a PPR and functions in pathogen recognition and internalization by binding and scavenging unwanted glycoprotein and glycolipids ${ }^{10,11}$. Notably, CD206 itself can bind polysaccharides from Streptococcus pneumoniae and lipopolysaccharide from Klebsiella pneumoniae subsequently promoting recognition, phagocytosis, and destruction of the bacterial cell ${ }^{10}$. Additionally, although detailed mechanisms underlying the proteolytic cleavage of CD206 are not fully understood, the shedding of CD206 from the membrane is enhanced upon the recognition of several pathogens ${ }^{18}$. The present study showed elevated sCD206 concentrations in accordance with CAP severity and the accumulation of CD206-positive macrophages in the lungs of fatal patients with CAP. These results suggested that increased sCD206 levels represent the activation of $\mathrm{CD} 206^{+}$macrophages during this disease and might partly result from macrophage-pathogen interactions.

A dichotomous approach to macrophage polarization is essential to understand the immune response. M1 macrophages are essential for anti-microbicidal and anti-tumor responses, whereas M2 macrophages have a central role in tissue repair and the resolution of inflammation ${ }^{19,20}$. CD206 is considered a marker of the M2 phenotype $^{6-9}$. Although the precise role of M2 macrophages in infectious disease with regard to complex M1/ M2 polarization remains unknown, increased expression of CD206 was previously reported in monocytes from patients with sepsis ${ }^{21,22}$. Additionally, the deletion of $\mathrm{CD}_{206^{+}}$macrophages exacerbates lung injury in endotoxemic mice ${ }^{23}$ and during Cryptococcus infection ${ }^{24}$. CD206 ${ }^{-1-}$ mice have been also reported to show increased allergic airway inflammation together with an elevated Th2/Th17 response $\mathrm{e}^{25}$. This suggests a protective role for M2 macrophages and sCD206 by inhibiting excessive inflammation.

It is well established that PSI and CURB65 predict mortality of CAP patients with high sensitivity and specificity. Meanwhile, it has been reported that these indicators might not be accurate for elderly patients with CAP. As atypical clinical presentations are usually found in elderly patients with CAP, the cut-off values for the scoring system could prove unreliable in these patients ${ }^{14-16}$. Although comorbidities are more frequent and the relative risk of mortality is higher in elderly patients, there is also a risk of the misclassification of younger patients with severe disease into lower classes. We previously reported that modified PSI together with performance status can predict mortality more accurately than conventional PSI in patients with CAP $\geq 80$ years of age ${ }^{15}$. Therefore, there is a need for age-independent surrogate markers to assess the severity of CAP. In this study, we demonstrated that sCD206 has the potential to predict mortality in patients with CAP, with a higher AUC value than that obtained 
(A)

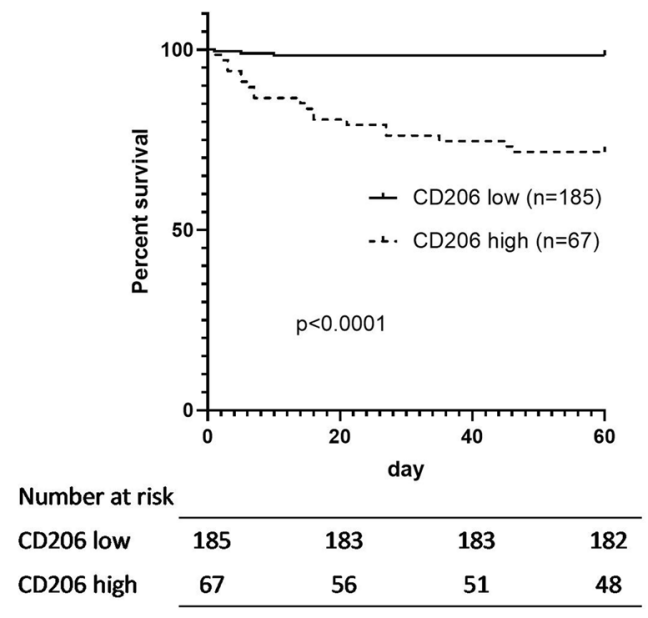

(B)

$<75 \mathrm{yr}$

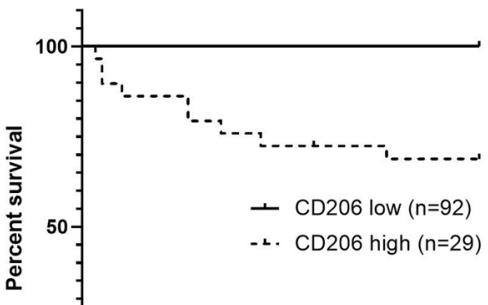

$p<0.0001$

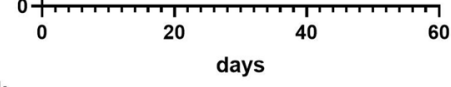

Number at risk

\begin{tabular}{lllll}
\cline { 2 - 4 } CD206 low & 92 & 92 & 92 & 92 \\
CD206 high & 29 & 25 & 21 & 19
\end{tabular}

(C)

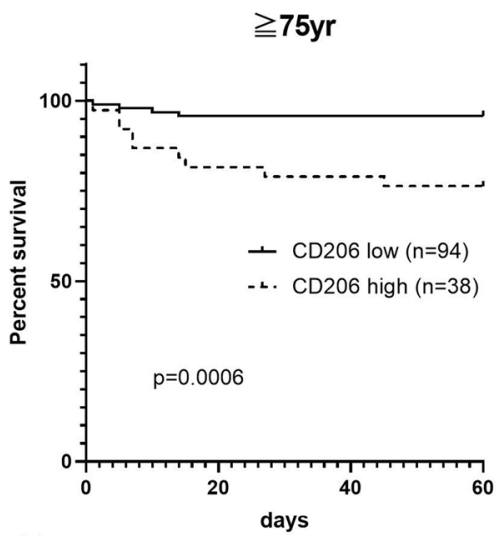

Number at risk

\begin{tabular}{lllll}
\cline { 2 - 4 } CD206 low & 94 & 91 & 91 & 90 \\
CD206 high & 38 & 32 & 31 & 29 \\
\hline
\end{tabular}

Figure 5. Kaplan-Meier curves of patients with community-acquired pneumonia (CAP). Kaplan-Meier curves of patients with CAP produced according to serum sCD206 concentrations. Kaplan-Meier curves of patients with CAP (A), CAP age $<75 \mathrm{yr}(\mathbf{B})$ and $\geq 75 \mathrm{yr}(\mathbf{C})$ according to serum sCD206 concentrations. P-values were determined by the log-rank test.

\begin{tabular}{|l|l|l|c|l|l|l|l|}
\hline \multirow{2}{*}{ Predictor } & HR & 95\% CI & p-value & HR & 95\% CI & $p$-value \\
\cline { 2 - 8 } & Univariate analysis & \multicolumn{2}{l|}{ Age and Gender adjusted Multivariate analysis } \\
\hline Sex, M/F & 1.12 & $0.454-2.790$ & 0.7990 & & & \\
\hline Age, years & 1.02 & $0.987-1.060$ & 0.2060 & & & $1.010-1.040$ & $<0.0001$ \\
\hline PSI (score) & 1.030 & $1.010-1.0400$ & $<0.0001$ & 1.030 & $1.540-3.460$ & $<0.0001$ \\
\hline CURB65 (score) & 2.31 & $1.560-3.430$ & $<0.0001$ & 2.310 & $1.500-10.30$ & 0.0053 \\
\hline Confusion & 4.10 & $1.580-10.70$ & 0.0038 & 3.930 & $2.81-36.90$ & 0.0004 \\
\hline Bacteremia & 8.93 & $2.650-30.20$ & 0.0004 & 10.20 & $1.000-1.040$ & 0.0108 \\
\hline BUN & 1.02 & $1.010-1.040$ & 0.0071 & 1.020 & $1.030-1.770$ & 0.0311 \\
\hline Cre & 1.34 & $1.030-1.75$ & 0.0318 & 1.350 & $0.100-0.565$ & 0.0012 \\
\hline Alb & 0.24 & $0.103-0.56$ & 0.0010 & 0.237 & $1.020-1.100$ & 0.0032 \\
\hline CRP & 1.06 & $1.020-1.100$ & 0.0031 & 1.060 & $0.990-0.998$ & 0.0034 \\
\hline P/F ratio & 0.994 & $0.990-0.998$ & 0.0027 & 0.994 & $1.120-1.270$ & $<0.0001$ \\
\hline CD206 (/100 ng/ml) & 1.190 & $1.120-1.270$ & $<0.0001$ & 1.190 & & \\
\hline
\end{tabular}

Table 3. Prediction of mortality: Logistic regression analysis. Prediction of mortality with community-acquired pneumonia patients: univariate and multivariate analyses. Multivariate analysis was adjusted by sex and age. HR; hazard ratio, CI; confidence interval, BUN; blood urea nitrogen, Cre; creatinine, Alb; albumin, CRP; C-reactive protein, $\mathrm{P} / \mathrm{F}$ ratio; $\mathrm{PaO}_{2} / \mathrm{FiO}_{2}$ ratio. 


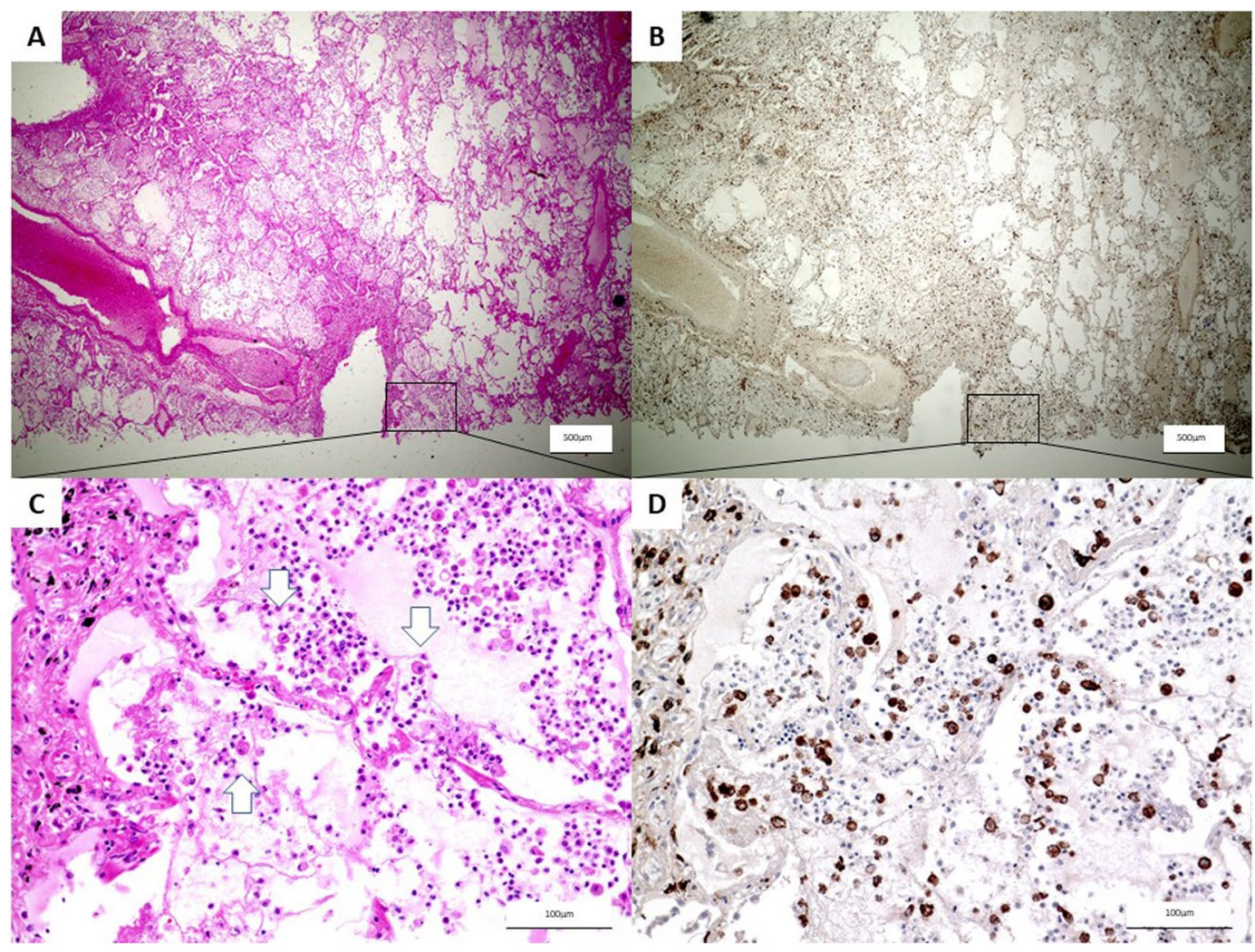

Figure 6. Immunohistochemical staining of CD206 in autopsied lungs of community-acquired pneumonia. 75 -year-old male autopsy case, patient was dead in two days from the onset of pneumonia. $(\mathbf{A} \times 20, \mathbf{C} \times 200) \mathrm{HE}$ stain shows strong infiltration of inflammatory cells and macrophages (arrow) and pulmonary congestion in alveolar. $(\mathbf{B} \times 20, \mathbf{D} \times 200)$ CD206 positive macrophages were observed in immunohistochemical staining.

for PSI. Additionally, sCD206 values were consistent in both age groups assessed, specifically, $<75$ years and $\geq 75$ years, suggesting that this approach could complement PSI or CURB65 assessments.

To date, in line with our study on CD206, the utility of macrophage-related markers such as scavenger receptor CD163 and the immunomodulatory molecule IDO have been vigorously investigated in clinical settings with a variety of diseases, as a surrogate marker to estimate disease severity and/or prognosis ${ }^{4,5,26-30}$. In addition, elevated levels of sCD206 in patients with interstitial lung disease ${ }^{31}$, critical illness ${ }^{32}$, and pneumococcal bacteremia ${ }^{33}$ were also reported. Therefore, elevated levels of these molecules including sCD206 are not disease-specific but might also comprehensively represent a whole-body immune response.

The present study had several limitations. First of all, although we examined two independent cohorts of CAP patients, the sample size was still inadequate and univariate and multivariate analyses were not performed with each cohort separately, thus a further validation study is necessary. Therefore, the absolute significance of sCD206 in CAP could not be determined. Second, the results clearly indicate that increased sCD206 is observed in patients with CAP; however, the origin of SCD206- and CD206-expressing monocytes was not investigated. In addition, the role of increased sCD206 levels in the etiology of infectious diseases is not completely elucidated.

\section{Conclusion}

In conclusion, the present study demonstrated elevated levels of serum sCD206 in patients with CAP, which is related to disease severity and clinical outcome. Furthermore, the SCD206 was useful for predicting outcome even in younger patients with CAP as well as elderly those with CAP. Our results suggested that sCD206 could be utilized as a novel prognostic marker for CAP and will help for better understanding the role of macrophage polarization in infectious disease.

\section{Methods}

Subjects. This prospective study was conducted using two cohorts of patients who were administered for the treatment of CAP in Hamamatsu, Japan. A cohort of 127 consecutive CAP patients admitted to Seirei-Mikatahara Hospital and Hamamatsu University School of Medicine between January 2007 and December 2010, and a cohort of 125 consecutive CAP patients hospitalized at Hamamatsu University School of Medicine between March 2013 and February 2018, were enrolled in this study. The former and latter cohorts were evaluated as a discovery cohort and validation cohort, respectively. Pneumonia was diagnosed according to previously published international guidelines ${ }^{34,35}$. CAP was defined as pneumonia that did not fulfill the criteria of hospital- or nursing and health care-acquired pneumonia in patients with symptoms of acute-onset lower respiratory tract infection, 
who demonstrated new infiltration on a chest radiograph. All patients were stratified into risk classes using the validated prediction rule, calculated according to the PSI and CURB-65 calculator (covering confusion, urea nitrogen, respiratory rate, and blood pressure, $\geq 65$ years of age $)^{12,13}$.

This study also included sera from 42 age and sex-matched subjects ( 30 men and 12 women, mean age of 73 years) who visited Hamamatsu University Hospital for health checks, as a control group. None of the control subjects had pulmonary infectious disease, as assessed by chest radiographs. This study was approved by the ethics committees of Hamamatsu University School of Medicine, Seirei-Mikatahara Hospital (15-167), and was carried out in accordance with approved guidelines. Written informed consent was obtained from all subjects in accordance with institutional guidelines. The study was registered in the University Hospital Medical Information Network in Japan (http://www.umin.ac.jp/. UMIN000003400 and UMIN000019472).

Sample collection. Blood samples were drawn at the time of admission before the start of empirical treatment. Serum samples were frozen at $-80^{\circ} \mathrm{C}$ until analysis; routine laboratory examinations such as blood cell counts and biochemical analyses were subsequently performed. The serum concentration of sCD206 was determined using an enzyme-linked immunosorbent assay (ELISA) kit (Ray Biotech, Norcross, GA, USA).

Immunohistochemistry. Lung biopsy specimens were obtained from autopsy cases, in which the patient died of severe pneumonia, and resected lung from a patient with early lung cancer was also examined. Tissues were fixed in $10 \%$ formalin and embedded in paraffin. Deparaffinized sections ( 5 - $\mu \mathrm{m}$-thick) were immersed in epitope retrieval solution (Target Retrieval Solution S1700; Dako North America, Inc., Carpinteria, CA, USA) and preheated at $120^{\circ} \mathrm{C}$ for $10 \mathrm{~min}$. After blocking endogenous peroxidase activity with $3 \% \mathrm{H}_{2} \mathrm{O}_{2}$ for 15 min, slides were incubated overnight with a mouse anti-human CD206 monoclonal antibody $(15 \mu \mathrm{g} / \mathrm{ml} ; \mathrm{R} \& \mathrm{D}$ Systems, Minneapolis, MN, USA) or IgG2b at $4{ }^{\circ} \mathrm{C}$. Subsequently, sections were incubated with visualization reagent (ChemMate Envision kit; Dako Japan, Inc., Tokyo, Japan) for $30 \mathrm{~min}$, followed by counterstaining with hematoxylin.

Statistical analysis. Discrete variables are expressed as counts (percentage), and continuous variables are expressed as the median [range] unless otherwise specified. The Mann-Whitney test was used for continuous variables. Categorical data were compared between groups by using the Fisher's exact test for independence. Correlations between sCD206 and clinical parameters were analyzed by the Spearman's rank correlation method. Overall survival time was measured from the date of CAP diagnosis. To examine the ability of sCD206 levels to predict mortality in patients with CAP, combined cohort data were analyzed. The areas under the receiver operating characteristic (ROC) curve were used to evaluate the ability of this marker to predict mortality. The optimal cut-off value of sCD206 in the combined cohort that ensured the best combinations of sensitivity and specificity was obtained. Cumulative survival probabilities were estimated by the Kaplan-Meier method with optimal cut-off values. The Log-rank test was used to compare survival among patients. Univariate and multivariate analyses were performed with Cox proportional hazards regression analysis with combined cohort subjects to predict mortality. Among the statistically significant covariates in the univariate analyses, several were excluded because of potential confounders and statistical limitations. Statistical analyses were performed using GraphPad Prism Version 8 (GraphPad Software, San Diego, CA, USA) and EZR (Saitama Medical Center, Jichi Medical University, Saitama, Japan), which is a graphical user interface for R (The R Foundation for Statistical Computing, Vienna, Austria). Statistical significance was considered at a P-value of 0.05 .

\section{Data availability}

The data are available from the authors upon reasonable request.

Received: 25 July 2019; Accepted: 27 November 2019;

Published online: 10 December 2019

\section{References}

1. World Health Organization. Media Center: The top 10 cause of death, https://www.who.int/news-room/fact-sheets/detail/the-top10-causes-of-death [accessed 2019 June 10].

2. Gordon, S. Alternative activation of macrophages. Nat Rev Immunol 3, 23-35, https://doi.org/10.1038/nri978 (2003).

3. Gordon, S. \& Martinez, F. O. Alternative activation of macrophages: mechanism and functions. Immunity 32, 593-604, https://doi. org/10.1016/j.immuni.2010.05.007 (2010).

4. Suzuki, Y. et al. Soluble hemoglobin scavenger receptor CD163 (sCD163) predicts mortality of community-acquired pneumonia. The Journal of infection 73, 375-377, https://doi.org/10.1016/j.jinf.2016.07.004 (2016).

5. Suzuki, Y. et al. Serum activity of indoleamine 2,3-dioxygenase predicts prognosis of community-acquired pneumonia. The Journal of infection 63, 215-222, https://doi.org/10.1016/j.jinf.2011.07.003 (2011).

6. Azad, A. K., Rajaram, M. V. \& Schlesinger, L. S. Exploitation of the Macrophage Mannose Receptor (CD206) in Infectious Disease Diagnostics and Therapeutics. J Cytol Mol Biol 1, 1-5, https://doi.org/10.13188/2325-4653.1000003 (2014).

7. Geijtenbeek, T. B. G. S. Signalling through C-type lectin receptors: shaping immune responses. Nat Rev Immunol 9, 465-479, https:// doi.org/10.1038/nri2569 (2009).

8. Hussell, T. \& Bell, T. J. Alveolar macrophages: plasticity in a tissue-specific context. Nat Rev Immunol 14, 81-93, https://doi. org/10.1038/nri3600 (2014).

9. Martinez-Pomares, L. The mannose receptor. J Leukoc Biol 92, 1177-1186, https://doi.org/10.1189/jlb.0512231 (2012).

10. Zamze, S. et al. Recognition of bacterial capsular polysaccharides and lipopolysaccharides by the macrophage mannose receptor. The Journal of biological chemistry 277, 41613-41623, https://doi.org/10.1074/jbc.M207057200 (2002).

11. Su, Y. et al. Glycosylation influences the lectin activities of the macrophage mannose receptor. The Journal of biological chemistry 280, 32811-32820, https://doi.org/10.1074/jbc.M503457200 (2005).

12. Fine, M. J. et al. A prediction rule to identify low-risk patients with community-acquired pneumonia. N Engl J Med 336, 243-250, https://doi.org/10.1056/nejm199701233360402 (1997) 
13. Lim, W. S. et al. Defining community acquired pneumonia severity on presentation to hospital: an international derivation and validation study. Thorax 58, 377-382, https://doi.org/10.1136/thorax.58.5.377 (2003).

14. Chen, J. H. et al. Comparison of clinical characteristics and performance of pneumonia severity score and CURB-65 among younger adults, elderly and very old subjects. Thorax 65, 971-977, https://doi.org/10.1136/thx.2009.129627 (2010).

15. Naito, T. et al. A validation and potential modification of the pneumonia severity index in elderly patients with community-acquired pneumonia. Journal of the American Geriatrics Society 54, 1212-1219, https://doi.org/10.1111/j.1532-5415.2006.00825.x (2006).

16. Parsonage, M., Nathwani, D., Davey, P. \& Barlow, G. Evaluation of the performance of CURB-65 with increasing age. Clinical microbiology and infection: the official publication of the European Society of Clinical Microbiology and Infectious Diseases 15, 858-864, https://doi.org/10.1111/j.1469-0691.2009.02908.x (2009).

17. Morales-Nebreda, L., Misharin, A. V., Perlman, H. \& Budinger, G. R. The heterogeneity of lung macrophages in the susceptibility to disease. Eur Respir Rev 24, 505-509, https://doi.org/10.1183/16000617.0031-2015 (2015).

18. Gazi, U. et al. Fungal recognition enhances mannose receptor shedding through dectin-1 engagement. The Journal of biological chemistry 286, 7822-7829, https://doi.org/10.1074/jbc.M110.185025 (2011).

19. Canton, J., Neculai, D. \& Grinstein, S. Scavenger receptors in homeostasis and immunity. Nat Rev Immunol 13, 621-634, https://doi. org $/ 10.1038 /$ nri3515 (2013)

20. Ka, M. B., Daumas, A., Textoris, J. \& Mege, J. L. Phenotypic diversity and emerging new tools to study macrophage activation in bacterial infectious diseases. Front Immunol 5, 500, https://doi.org/10.3389/fimmu.2014.00500 (2014).

21. Brunialti, M. K. et al. Increased percentages of T helper cells producing IL-17 and monocytes expressing markers of alternative activation in patients with sepsis. PLoS One 7, e37393, https://doi.org/10.1371/journal.pone.0037393 (2012).

22. Kjaergaard, A. G. et al. Monocyte expression and soluble levels of the haemoglobin receptor (CD163/sCD163) and the mannose receptor (MR/sMR) in septic and critically ill non-septic ICU patients. PLoS One 9, e92331, https://doi.org/10.1371/journal. pone.0092331 (2014).

23. Kambara, K. et al. In vivo depletion of CD206+ M2 macrophages exaggerates lung injury in endotoxemic mice. Am J Pathol 185, 162-171, https://doi.org/10.1016/j.ajpath.2014.09.005 (2015).

24. Dan, J. M., Kelly, R. M., Lee, C. K. \& Levitz, S. M. Role of the mannose receptor in a murine model of Cryptococcus neoformans infection. Infection and immunity 76, 2362-2367, https://doi.org/10.1128/iai.00095-08 (2008).

25. Zhou, Y. et al. Mannose receptor modulates macrophage polarization and allergic inflammation through miR-511-3p. The Journal of allergy and clinical immunology 141, 350-364 e358, https://doi.org/10.1016/j.jaci.2017.04.049 (2018).

26. Enomoto, Y. et al. Clinical significance of soluble CD163 in polymyositis-related or dermatomyositis-related interstitial lung disease. Arthritis research \& therapy 19, 9, https://doi.org/10.1186/s13075-016-1214-8 (2017).

27. Suzuki, Y. et al. Utility of Macrophage-activated Marker CD163 for Diagnosis and Prognosis in Pulmonary Tuberculosis. Annals of the American Thoracic Society 14, 57-64, https://doi.org/10.1513/AnnalsATS.201607-528OC (2017).

28. Suzuki, Y. et al. Macrophage mannose receptor, CD206, predict prognosis in patients with pulmonary tuberculosis. Scientific reports 8, https://doi.org/10.1038/s41598-018-31565-5 (2018).

29. Suzuki, Y. et al. Serum indoleamine 2,3-dioxygenase activity predicts prognosis of pulmonary tuberculosis. Clinical and vaccine immunology: CVI 19, 436-442, https://doi.org/10.1128/cvi.05402-11 (2012).

30. Suzuki, Y. et al. Increased serum kynurenine/tryptophan ratio correlates with disease progression in lung cancer. Lung cancer (Amsterdam, Netherlands) 67, 361-365, https://doi.org/10.1016/j.lungcan.2009.05.001 (2010).

31. Horiike, Y. et al. Successful classification of macrophage-mannose receptor CD206 in severity of anti-MDA5 antibody positive dermatomyositis associated ILD. Rheumatology 58, 2143-2152, https://doi.org/10.1093/rheumatology/kez185 (2019).

32. Rodgaard-Hansen, S. et al. A soluble form of the macrophage-related mannose receptor (MR/CD206) is present in human serum and elevated in critical illness. Clinical chemistry and laboratory medicine 52, 453-461, https://doi.org/10.1515/cclm-2013-0451 (2014).

33. Rodgaard-Hansen, S. et al. Increased concentrations of the soluble mannose receptor in serum from patients with pneumococcal bacteraemia, and prediction of survival. Infect Dis (Lond) 47, 203-208, https://doi.org/10.3109/00365548.2014.984321 (2015).

34. Guidelines for the management of adults with hospital-acquired, ventilator-associated, and healthcare-associated pneumonia. Am J Respir Crit Care Med 171, 388-416, https://doi.org/10.1164/rccm.200405-644ST (2005).

35. Mandell, L. A. et al. Infectious Diseases Society of America/American Thoracic Society consensus guidelines on the management of community-acquired pneumonia in adults. Clin Infect Dis 44(Suppl 2), S27-72, https://doi.org/10.1086/511159 (2007).

\section{Acknowledgements}

Y.S. was supported by a grant-in-aid for scientific research (19K17632) from the Japan Society for the Promotion of Science and scholarship grant for research in basic medical science and medical care (18KI050) from the Ichiro Kanehara Foundation for the Promotion of Medical Sciences and Medical Care. We thank Editage for editing a draft of this manuscript (supported by a grant-in-aid for scientific research from the Japan Society for the Promotion of Science and the Ichiro Kanehara Foundation). This work was supported by a grant-in-aid for scientific research (19K17632 to Y.S.) from the Japan Society for the Promotion of Science and scholarship grant for research in basic medical science and medical care (18KI050 to Y.S.) from the Ichiro Kanehara Foundation for the Promotion of Medical Sciences and Medical Care.

\section{Author contributions}

Kazuo Tsuchiya: Data collection, data analysis, CD206 staining, interpretation, and manuscript writing, Yuzo Suzuki: Concept and design, data analysis, and interpretation, manuscript writing, final approval of the manuscript; Katsuhiro Yoshimura: Data collection, Hideki Yasui: Data collection; Masato Karayama: Data collection; Hironao Hozumi: Data collection; Kazuki Furuhashi: Data collection; Noriyuki Enomoto: Data collection; Tomoyuki Fujisawa: Data collection, Yutaro Nakamura: Data collection; Naoki Inui: Data collection; Koshi Yokomura: Data collection; Takafumi Suda: Administrative support, data analysis and interpretation.

\section{Competing interests}

The authors declare no competing interests.

\section{Additional information}

Supplementary information is available for this paper at https://doi.org/10.1038/s41598-019-55289-2.

Correspondence and requests for materials should be addressed to Y.S.

Reprints and permissions information is available at www.nature.com/reprints. 
Publisher's note Springer Nature remains neutral with regard to jurisdictional claims in published maps and institutional affiliations.

(c) (i) Open Access This article is licensed under a Creative Commons Attribution 4.0 International License, which permits use, sharing, adaptation, distribution and reproduction in any medium or format, as long as you give appropriate credit to the original author(s) and the source, provide a link to the Creative Commons license, and indicate if changes were made. The images or other third party material in this article are included in the article's Creative Commons license, unless indicated otherwise in a credit line to the material. If material is not included in the article's Creative Commons license and your intended use is not permitted by statutory regulation or exceeds the permitted use, you will need to obtain permission directly from the copyright holder. To view a copy of this license, visit http://creativecommons.org/licenses/by/4.0/.

(c) The Author(s) 2019 